\title{
Robust waveform design for MIMO-STAP to improve the worst-case detection performance
}

\author{
Hongyan Wang ${ }^{1,2^{*}}$, Guisheng Liao ${ }^{1}$, Jun $\mathrm{Li}^{1}$ and Wangmei Guo ${ }^{3}$
}

\begin{abstract}
In this article, we address the problem of robust waveform optimization for improving the worst-case detection performance of multi-input multi-output (MIMO) space-time adaptive processing (STAP) in the presence of colored Gaussian disturbance. A novel diagonal loading-based method is proposed to optimize the waveform covariance matrix for maximizing the worst-case output signal-interference-noise ratio (SINR) over the convex uncertainty set such that the worst-case detection performance of MIMO-STAP can be maximized. The resultant nonlinear optimization problem is reformulated as a semidefinite programming problem, which can be solved very efficiently. Numerical examples show that the worst-case output SINR of MIMO-STAP can be improved considerably by the proposed method compared to that of uncorrelated waveforms.
\end{abstract}

Keywords: Multi-Input Multi-Output (MIMO) radar, Waveform optimization, Space-Time Adaptive Processing (STAP), Diagonal Loading (DL), SemiDefinite Programming (SDP)

\section{Introduction}

In recent years, multiple-input multiple-output (MIMO) techniques have received more and more attention from both the communication and radar communities [1-13]. MIMO radar can employ multiple transmitting elements to transmit arbitrary waveforms other than coherent waveforms in traditional phased-array radars. Two categories of MIMO radar systems can be classified by the configuration of the transmitting and receiving antennas: (1) MIMO radar with widely separated antennas (see e.g., [1]), and (2) MIMO radar with colocated antennas (see e.g., [2]). For MIMO radar with widely separated antennas, the transmitting and receiving elements are widely spaced such that each views a different aspect of the target. Similar to the multipath diversity concept in wireless communication over fading channels [13], this type of MIMO radar can exploit the spatial diversity to overcome the performance degradation caused by target scintillations [1]. In contrast, MIMO radar with colocated antennas, whose elements in transmitting and receiving arrays are close enough such that the target radar cross sections (RCSs)

\footnotetext{
*Correspondence: gglongs@163.com

${ }^{1}$ National Key Laboratory of Radar Signal Processing, Xidian University, Xi'an

710071, China

${ }^{2}$ No.20 Research Institute of China Electronics Technology Group

Corporation, Xi'an 710068, China

Full list of author information is available at the end of the article
}

observed by MIMO radar are identical, can utilize the waveform diversity to increase the virtual aperture of the receiving array [2]. Accordingly, it has several advantages including improved parameter identifiability $[3,4]$, and more flexibility for transmit beampattern design [5-7].

To improve the detection performance of MIMO radar, one way is detector design which was investigated in $[8,9]$. Chong et al. [8] proposed the constant false alarm rate generalized likelihood ratio test-linear quadratic (GLRTLQ) detector for MIMO radar in the scenario of nonGaussian clutter. He et al. [9] derived GLRT moving target detectors for centralized MIMO and distributed MIMO.

Another way to improve the detection performance of MIMO radar is waveform optimization, which has been studied in [6,7]. In [6], a gradient-based method is proposed to maximize the output signal-to-interference-plus-noise ratio (SINR) for improving the detection performance for extended target; unfortunately, it cannot guarantee nondecreasing SINR in each iteration step. In order to guarantee convergence, a new iterative algorithm is proposed in [7].

It is known that waveform optimization for improving the performance of MIMO radar usually depends on the initial parameter estimate (i.e., some prior information on the target of interest and scenario) [5-7]. In practice, these parameters are estimated with errors and hence must have uncertain. Therefore, the resultant performance of MIMO 
radar may be sensitive to the estimation errors and uncertainty in parameters (see e.g., [5]). It means that the optimized waveforms based on a certain parameter estimate can give a very poor performance for another reasonable estimate.

Space-time adaptive processing (STAP) technique plays an important role in numerous civilian and military applications such as surveillance, airborne moving target indication (MTI), and ground MTI [14]. The basic theory of STAP for traditional phased-array radar has been well developed [15]. The concept of MIMO-STAP is proposed in [10]. A new algorithm for MIMO-STAP with orthogonal waveforms is proposed in [11], which can significantly lower the computational complexity compared to fully adaptive methods. Under the general waveform assumption, the relationship between the clutter rank of MIMOSTAP and the transmitted waveforms has profoundly been studied in [12].

In this article, we consider the problem of robust waveform design in the presence of colored Gaussian disturbance (including clutter, jamming, and thermal noise), which maximizes the worst-case detection performance of MIMO-STAP. Because maximization of the output SINR is tantamount to maximization of the detection performance in the case of Gaussian noise (see e.g., [6,7] and the references therein for more details), here the waveform covariance matrix (WCM) is optimized to maximize the worst-case output SINR of MIMO-STAP over the convex uncertainty set such that the worst-case detection performance can be maximized. The waveform design is formulated in terms of a rather complicated nonlinear optimization problem. Consequently, this problem cannot be easily solved by convex optimization method. A novel diagonal loading (DL)-based method [16] is proposed to formulate the resultant optimization problem as a semidefinite programming (SDP) problem [17], which can be solved very efficiently.

The remainder of this article is organized as follows. The MIMO-STAP model is introduced, and the robust optimization problem is formulated in Section 2. A novel DL-based method is proposed to formulate the resultant nonlinear optimization problem as an SDP in Section 3. The effectiveness of the proposed method is verified via numerical examples in Section 4. Finally, conclusions are given in Section 5.

Throughout the article, matrices and vectors are denoted by boldface uppercase and lowercase letters, respectively. The character I denotes the identity matrix, $\operatorname{tr}(\cdot)$ indicates the trace of a matrix, and $\otimes$ indicates the Kronecker product. vec(.) is the vectorization operator stacking the columns of a matrix on top of each other. We use $(.)^{T},(.)^{*}$, and $(.)^{H}$ to denote, respectively, the transpose, conjugate, and conjugate transpose. The notation $A \preccurlyeq B$ means that $\mathbf{B}-\mathbf{A}$ is positive semidefinite.

\section{Problem formulation}

The MIMO-STAP signal model adopted in this article is similar to that developed in [11]. The only difference is that the transmitted waveforms other than the receiving weight vector are considered here. For the MIMO radar exploited here, there are $M$ isotropic transmitting elements with uniform space $d_{T}$, and $N$ receiving elements with uniform space $d_{R}$. At each transmitting element, a coherent processing interval (CPI) consists of a burst of $L$ pulses with a constant pulse repetition interval (PRI) $T$. For the $n$th receiving element, the received signal in the $l$ th PRI can be expressed as

$$
\begin{aligned}
\mathbf{y}_{n, l}= & \sum_{m=0}^{M-1} \rho_{t} \mathbf{s}_{m}^{T} e^{j \frac{2 \pi}{\lambda}\left(\sin \theta_{t}\left(d_{R} n+d_{T} m+2 v T l\right)+2 v_{t} T l\right)} \\
& +\int_{\theta=0}^{2 \pi} \sum_{m=0}^{M-1} \rho(\theta) \mathbf{s}_{m}^{T} e^{j \frac{2 \pi}{\lambda}\left(\sin \theta\left(d_{R} n+d_{T} m+2 v T l\right)\right)} d \theta \\
& +\mathbf{z}_{n, l},
\end{aligned}
$$

where $s_{m} \in \mathrm{C}^{K \times 1}$ is the discrete version of the complex baseband signal with $K$ snapshots transmitted by the $m$ th transmitting element in each PRI. $\rho_{\mathrm{t}}$ and $\theta_{t}$ represent, respectively, the complex amplitude and location of the target at the considered range bin. $v$ and $v_{t}$ denote the speed of the radar station and the target speed toward MIMO radar, respectively. $\rho(\theta)$ is the reflect coefficient of clutter patch at $\theta$, and $\lambda$ is the operation wavelength. The term $\mathbf{z}_{n}$, ${ }_{l}$ denotes the interference plus noise received by the $n$th receiving element in the $l$ th PRI.

Based on the signal model shown in (1), the output SINR is derived in Appendix 1 and given by

$$
\operatorname{SINR}=\left|\rho_{t}\right|^{2} \mathbf{v}_{t}^{H}\left(\mathbf{I}_{\mathrm{MNL}}+\mathbf{R}_{\mathrm{TS}} \mathbf{R}_{\mathrm{C}}\right)^{-1} \mathbf{R}_{\mathrm{TS}} \mathbf{v}_{t},
$$

where $\mathbf{v}_{\mathrm{t}}, \mathbf{R}_{\mathrm{C}}, \mathbf{R}_{\mathrm{TS}}$ are given in (24) and (25), respectively.

Obviously, the calculation of the SINR in (2) requires the specification of $\mathbf{v}_{\mathrm{t}}$, the clutter covariance matrix, and the noise plus interference term, i.e., $\theta_{t}, f_{\mathrm{D}}, \mathbf{R}_{\mathrm{C}}$, and $\mathbf{Q}$. As a sequence, waveform optimization for maximizing the SINR explicitly depends on these pre-assigned values. In practice, these parameters are estimated with errors and so they are uncertain. Therefore, the resultant detection performance of MIMO-STAP via waveform optimization may be sensitive to estimation errors and uncertainty in parameters. In this article, we only consider the effect on the output SINR performance of the estimation error of the spatial-temporal vector, i.e., $\mathbf{v}_{t}$.

We assume that $\mathbf{v}_{t}$ is uncertain, but known to belong to a convex compact set, which is modeled in Appendix 2, and can be illustrated as 


$$
V=\left\{\widetilde{\mathrm{v}}_{t} \mid \eta \leq\left\|\widetilde{\mathrm{v}}_{t}\right\|\right\}
$$

where $\widetilde{\mathbf{v}}_{t}=\mathbf{v}_{t}+\boldsymbol{\sigma}$, in which $\widetilde{\mathbf{v}}_{t}$ and $\mathbf{v}_{t}$ are, respectively, the actual and corresponding presumed spatial-temporal vectors. $\boldsymbol{\sigma}$ denotes the unknown complex vectors describing errors of $\widetilde{\mathbf{v}}_{t}$, and $\eta$ is the lower bound of the norm of $\widetilde{\mathbf{v}}_{t}$, which is shown in (34).

The robust waveform optimization for improving the worst-case detection performance of MIMO-STAP can now be briefly stated as follows: Optimize the WCM to maximize the worst-case output SINR over the convex set $\mathbf{V}$ under the total power constraint on $\mathbf{R}_{\mathrm{S}}$, which can be formulated as

$$
\begin{array}{ccl}
\max _{\mathbf{R}_{\mathrm{S}}} \min _{\widetilde{v}_{t}} & \widetilde{\mathbf{v}}_{t}^{H}\left(\mathbf{I}_{\mathrm{MNL}}+\mathbf{R}_{\mathrm{TS}} \mathbf{R}_{\mathrm{C}}\right)^{-1} \mathbf{R}_{\mathrm{TS}} \widetilde{\mathbf{v}}_{t} \\
\text { s.t. } & \widetilde{\mathbf{v}}_{t} \in \mathbf{V} \\
& \operatorname{tr}\left(\mathbf{R}_{\mathrm{S}}\right)=K P \\
& \mathbf{R}_{\mathrm{S}} \succeq 0
\end{array}
$$

where $P$ denotes the total transmitted power.

The worst-case will happen when $\widetilde{\mathbf{v}}_{t}$ is in the direction of eigenvector corresponding to the smallest eigenvalue of $\left(I_{\mathrm{MNL}}+R_{T S} R_{C}\right)^{-1} R_{T S}$. Therefore, with (3), (4) can be written as

$$
\begin{array}{ll}
\max _{\mathbf{R}_{\mathrm{S}}} & \eta^{2} \lambda_{\min }\left(\left(\mathbf{I}_{\mathrm{MNL}}+\mathbf{R}_{\mathrm{TS}} \mathbf{R}_{\mathrm{C}}\right)^{-1} \mathbf{R}_{\mathrm{TS}}\right) \\
\text { s.t. } & \operatorname{tr}\left(\mathbf{R}_{\mathrm{S}}\right)=K P \\
& \mathbf{R}_{\mathrm{S}} \succeq \mathbf{0}
\end{array}
$$

where $\lambda_{\text {min }}(\cdot)$ denotes the smallest eigenvalue of a matrix.

It can be seen that the problem in (5) is a rather complicated nonlinear function of $\mathbf{R}_{\mathrm{S}}$ due to $R_{T S} \geqslant 0$ and $R_{C} \geqslant 0$. Hence, the problem is difficult to be solved by convex optimization method [17].

\section{Solution to the optimization problem}

In this section, we demonstrate how to obtain an optimal solution of the nonlinear optimization problem in (5). For this purpose, the DL approach, which has commonly been exploited in the robust beamforming (see e.g., [16]), is employed to $\mathbf{R}_{\mathbf{S}}$ such that

$$
\widetilde{\mathbf{R}}_{\mathrm{S}}=\mathbf{R}_{\mathrm{S}}+\rho \mathbf{I} \succ 0,
$$

where $\rho \ll \lambda_{\max }\left(\mathbf{R}_{\mathrm{S}}\right), \lambda_{\max }(\cdot)$ is the largest eigenvalue of a matrix. Note that $\widetilde{\mathbf{R}}_{\mathrm{TS}}=\mathbf{I}_{L} \otimes \widetilde{\mathbf{R}}_{S}^{T} \otimes \mathbf{Q}^{-1} \succ 0$ due to $\widetilde{\mathbf{R}}_{S} \succ 0$ [18]. By replacing $\mathbf{R}_{\mathrm{TS}}$ in (5) with $\widetilde{\mathbf{R}}_{\mathrm{TS}}$, we can obtain

$$
\begin{array}{ll}
\max _{\mathbf{R}_{\mathrm{S}}} & \eta^{2} \lambda_{\min }\left(\left(\mathbf{I}_{\mathrm{MNL}}+\widetilde{\mathbf{R}}_{\mathrm{TS}} \mathbf{R}_{\mathrm{C}}\right)^{-1} \widetilde{\mathbf{R}}_{\mathrm{TS}}\right) \\
\text { s.t. } & \operatorname{tr}\left(\mathbf{R}_{\mathrm{S}}\right)=K P \\
& \mathbf{R}_{\mathrm{S}} \succeq \mathbf{0}
\end{array}
$$

Because $\widetilde{\mathbf{R}}_{\mathrm{TS}} \succ \mathbf{0}$, (7) can be reformulated as [18]

$$
\begin{array}{ll}
\max _{\mathbf{R}_{\mathrm{S}}} & \eta^{2} \lambda_{\min }\left(\left(\widetilde{\mathbf{R}}_{\mathrm{TS}}^{-1}+\mathbf{R}_{\mathrm{C}}\right)^{-1}\right) \\
\text { s.t. } & \operatorname{tr}\left(\mathbf{R}_{\mathrm{S}}\right)=K P \\
& \mathbf{R}_{\mathrm{S}} \succeq \mathbf{0}
\end{array}
$$

By using the matrix inversion lemma [18], (8) can be reshaped as

$$
\begin{array}{ll}
\max _{\mathbf{R}_{\mathrm{S}}} & \eta^{2} \lambda_{\max }\left(\widetilde{\mathbf{R}}_{\mathrm{TS}}^{-1}+\mathbf{R}_{\mathrm{C}}\right) \\
\text { s.t. } & \operatorname{tr}\left(\mathbf{R}_{\mathrm{S}}\right)=K P \\
& \mathbf{R}_{\mathrm{S}} \succeq \mathbf{0}
\end{array}
$$

According to [19], (9) can be represented as a solution to the problem

$$
\begin{array}{ll}
\min _{\mathbf{R}_{\mathrm{S}}, t} & \eta^{2} t \\
\text { s.t. } & \widetilde{\mathbf{R}}_{\mathrm{TS}}^{-1}+\mathbf{R}_{\mathrm{C}} \preccurlyeq t \mathbf{I}, \\
& \operatorname{tr}\left(\mathbf{R}_{\mathrm{S}}\right)=K P \\
& \mathbf{R}_{\mathrm{S}} \succeq \mathbf{0}
\end{array}
$$

where $t$ is an auxiliary variable.

The problem (10) can be recast as an SDP relying on the following lemma [20, pp. 472].

Lemma 1 (Schur's complement): Let $Z=\left[\begin{array}{cc}A & B^{H} \\ B & C\end{array}\right]$ be a Hermitian matrix with $C \succ 0$, then $Z \geqslant 0$ if and only if $\Delta \mathbf{C} \geqslant 0$, where $\Delta \mathbf{C}$ is the Schur complement of $\mathbf{C}$ in $\mathbf{Z}$ and is given by $\Delta \mathrm{C}=A-B^{H} C^{-1} B$.

By using Lemma 1, (10) can readily be reformulated as an SDP

$$
\begin{array}{ll}
\min _{\mathbf{R}_{\mathrm{S}}, t} & \eta^{2} t \\
\text { s.t. } & {\left[\begin{array}{cc}
t \mathbf{I}-\mathbf{R}_{\mathrm{C}} & \mathbf{I} \\
\mathbf{I} & \widetilde{\mathbf{R}}_{\mathrm{TS}}
\end{array}\right] \succcurlyeq \mathbf{0 .}} \\
& \operatorname{tr}\left(\mathbf{R}_{\mathrm{S}}\right)=K P \\
& \mathbf{R}_{\mathrm{S}} \succeq \mathbf{0}
\end{array}
$$

\subsection{Remark}

As illustrated in [21], the main shortcoming of the DL approach is that it is not clear how to obtain the optimal value of the DL factor $\rho$. Hence, this problem is still an open problem required to be investigated in the future. In the following, we choose $\rho=K P / 1000$ by numerical examples.

Using many well-known algorithms for solving SDP problems [17], (11) can be solved very efficiently. In the 
following examples, the optimization toolbox in [22] is used for the problem. Note that we only obtain the WCM other than the ultimate transmitted waveforms in this article. In practice, the ultimate waveforms can asymptotically be synthesized by using the method in [23].

\section{Numerical examples}

In this section, we assess the SINR performance of the proposed method compared to that of uncorrelated waveforms which can be generated by using Hadamard codes [13].

The basic parameters for the following examples are $M=3, N=3, L=3, \beta=1, v=200 \mathrm{~m} / \mathrm{s}, f_{\mathrm{D}}=0.0649$, and $K=256$. The altitude is $9 \mathrm{~km}$, and the range of interest is $12.728 \mathrm{~km}$. We use the following two MIMO radar systems with various antenna configurations: MIMO radar $(0.5,0.5)$, i.e., $\gamma=1$, and MIMO radar $(1.5,0.5)$, i.e., $\gamma=3$, where the parameters specifying each radar system are the inter-element spacing of the transmitter and receiver (in units of wavelengths), respectively. The array signal-to-noise ratio (ASNR) varying from 10 to $50 \mathrm{~dB}$ in the following examples is defined as $\mathrm{PMN} / \sigma_{W}^{2}$, where $\sigma_{W}^{2}$ denotes the variance of the additive white thermal noise. One target with unit amplitude at $-4^{\circ}$ is considered. The clutter is modeled using discrete points, the RCSs for which are modeled as identical independent Gaussian random variables with mean zero and variance $\sigma_{i}^{2}, i=1, \ldots, N_{C}$, and assumed to be fixed in the CPI. The sample points are equally spaced on the range bin, and the number of clutter points $N_{C}$ is 10,000 . The clutter-to-noise ratio (CNR) varies from 10 to $50 \mathrm{~dB}$. There are two jammers located at $15^{\circ}$ and $-20^{\circ}$. The jammer-to-noise ratio for each jammer is $60 \mathrm{~dB}$. The jammers are modeled as point sources which transmit independent white Gaussian signals uncorrelated with the signals transmitted by MIMO radar.

In the following examples, we assume that the initial angle and normalized Doppler frequency estimate errors have uncertainties $\Delta \theta=\left[-3^{\circ}, 3^{\circ}\right]$ and $\Delta f_{D}=[-0.04,0.04]$, respectively. It means that $\theta$ " belongs to $\left[-7^{\circ},-1^{\circ}\right]$ and $f^{\prime \prime}{ }_{D}$ belongs to $[0.0249,0.1049]$, where $\theta$ " and $f^{\prime \prime}{ }_{D}$ denote, respectively, the estimate of $\theta$ and $f_{D}$. After calculating, we can obtain $\boldsymbol{\sigma}=4.1477$ and $\eta=1.0484$ for MIMO radar $(0.5,0.5)$, as well as $\boldsymbol{\sigma}=5.1398$ and $\eta=0.9436$ for the other case.

Figure 1 shows the optimal transmit beampatterns optimized by the proposed method in the case of ASNR = $30 \mathrm{~dB}$ and $\mathrm{CNR}=30 \mathrm{~dB}$. One can observe that the proposed method places a peak around the target location, that is, the worst-case detection performance in the convex uncertainty may be improved. Moreover, we can see grating lobes of the peak in the case of MIMO radar $(1.5,0.5)$ shown in Figure $1 \mathrm{~b}$, which is due to the sparse transmitting array.

The worst-case output SINRs obtained by using our method and uncorrelated waveforms are compared in Figure 2 as a function of ASNR or CNR. It can be seen that the worst-case SINR obtained by the proposed method or uncorrelated waveform increases as the increasing of ASNR, while decreases as the increasing of CNR. Moreover, the proposed method can significantly improve the worst-case SINR performance compared to uncorrelated waveforms, regardless of ASNR or CNR. Furthermore, comparing Figure $2 \mathrm{a}$ with $\mathrm{b}$ or $\mathrm{c}$ with $\mathrm{d}$, one can see that the SINR for MIMO radar $(1.5,0.5)$ is
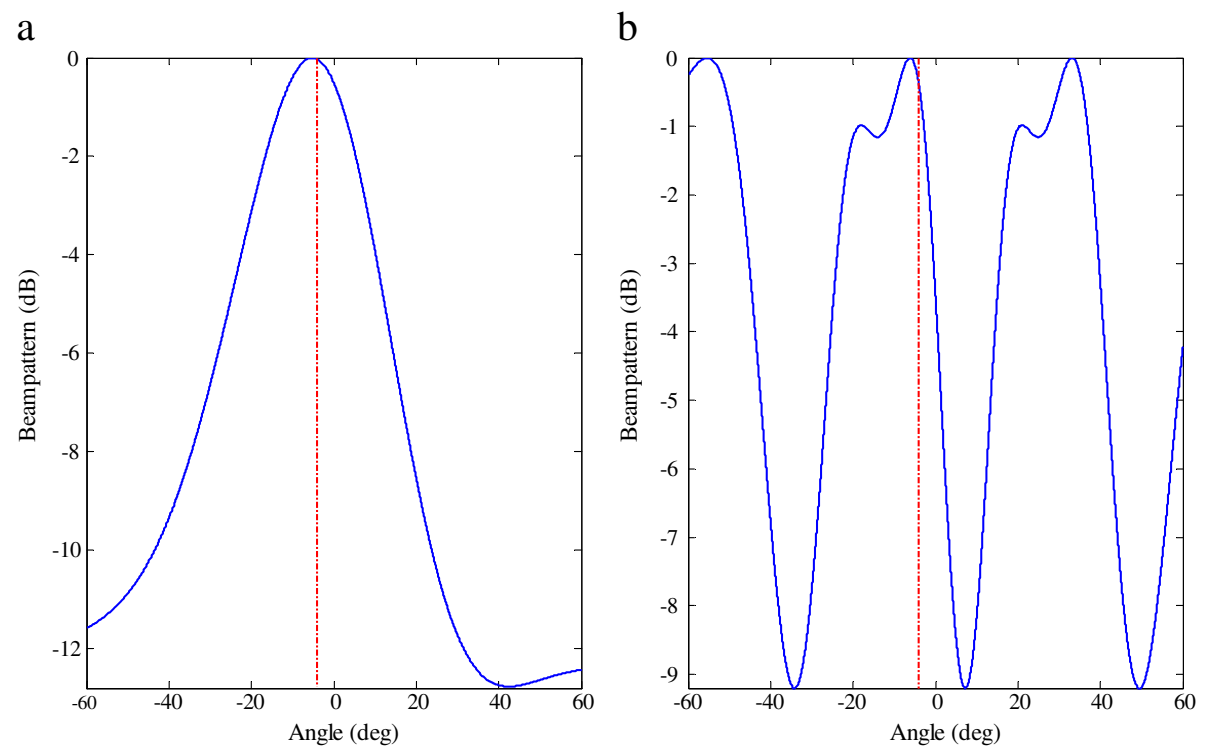

Figure 1 Optimal transmit beampatterns obtained by the proposed method with $A S N R=30 \mathrm{~dB}$ and $\mathrm{CNR}=\mathbf{3 0} \mathrm{dB}$. (a) Optimal transmit beampatterns for MIMO radar $(0.5,0.5)$. (b) Optimal transmit beampatterns for MIMO radar $(1.5,0.5)$. 

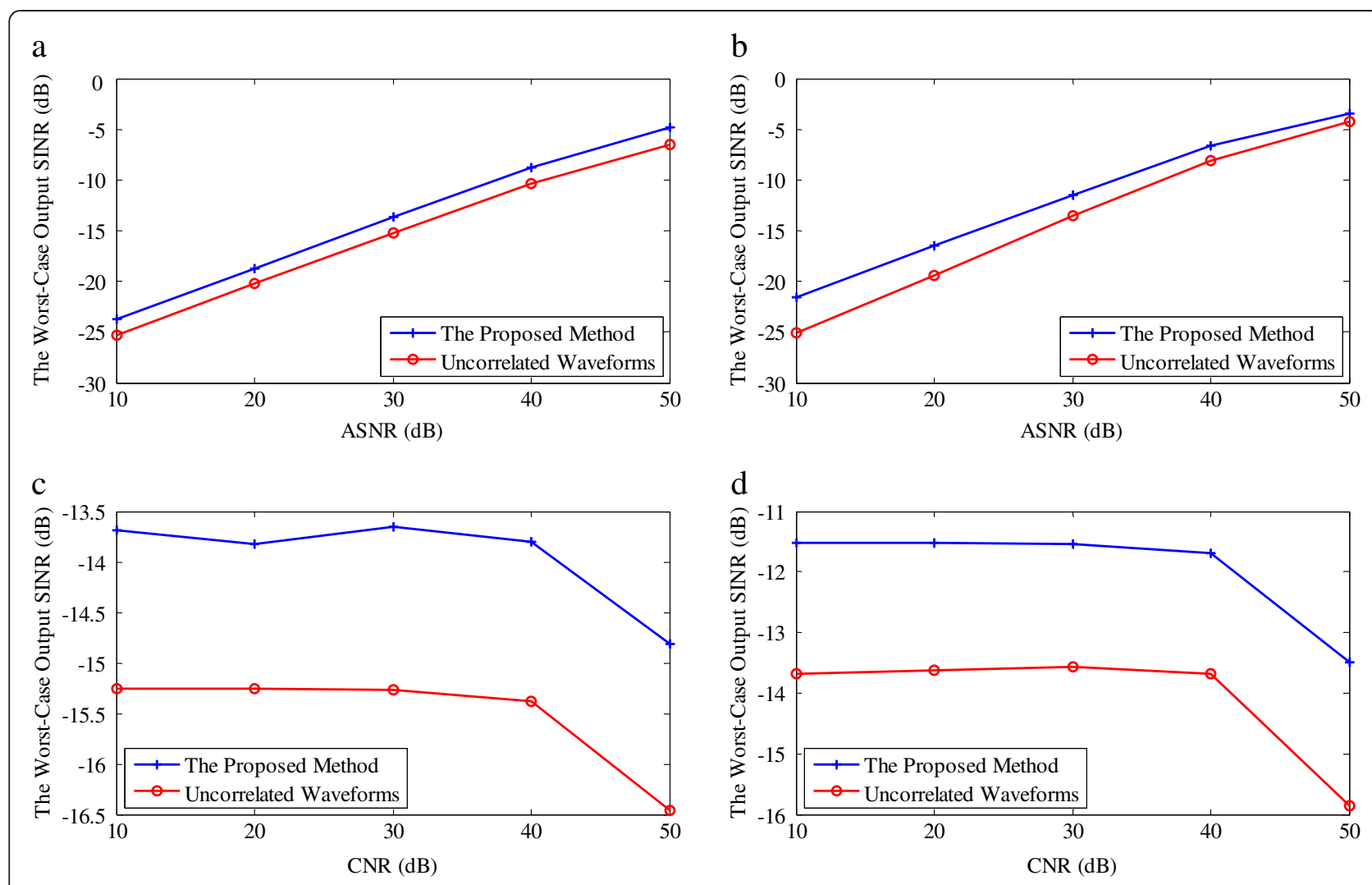

Figure 2 The worst-case output SINR obtained by the proposed method as a function of ASNR or CNR, as well as that of uncorrelated waveforms. (a) SINR versus ASNR with $C N R=30 \mathrm{~dB}$ for MIMO radar $(0.5,0.5)$. (b) SINR versus ASNR with $C N R=30 \mathrm{~dB}$ for MIMO radar $(1.5,0.5)$. (c) SINR versus CNR with $A S N R=30 \mathrm{~dB}$ for MIMO radar $(0.5,0.5)$. (d) SINR versus CNR with $A S N R=30 \mathrm{~dB}$ for MIMO radar $(1.5,0.5)$.

larger than that of MIMO radar $(0.5,0.5)$, which is due to that the virtual receiving array aperture for the former is much larger than that for the latter [11].

\section{Conclusions}

In this article, we have investigated the problem of robust waveform optimization for improving the worst-case detection performance of MIMO-STAP by explicitly incorporating the parameter estimate uncertainty into the optimization model. A novel DL-based method has been proposed to maximize the worst-case output SINR of MIMO-STAP such that the worst-case detection performance can be maximized. The proposed method can reformulate the resultant nonlinear optimization problem as an SDP problem, which can be solved very efficiently. Numerical examples have shown that the proposed method can significantly improve the worst-case output SINR compared to uncorrelated waveforms.

\section{Appendix 1}

If the isorange ring is divided in the cross-range dimension into $N_{C}\left(N_{C} \gg \mathrm{NML}\right)$ clutter patches, then (1) can be rewritten as

$$
\begin{aligned}
\mathbf{y}_{n, l}= & \sum_{m=0}^{M-1} \rho_{t} \mathbf{s}_{m}^{T} e^{j \frac{2 \pi}{\pi}\left(\sin \theta_{t}\left(d_{R} n+d_{T} m+2 v T l\right)+2 v_{t} T l\right)} \\
& +\sum_{i=0}^{N_{C}-1} \sum_{m=0}^{M-1} \rho\left(\theta_{i}\right) \mathbf{s}_{m}^{T} e^{j \frac{2 \pi}{\lambda}\left(\sin \theta_{i}\left(d_{R} n+d_{T} m+2 v T l\right)\right)} \\
& +\mathbf{z}_{n, l}
\end{aligned}
$$

Define $f_{s}=\frac{d_{R} \sin \theta_{t}}{\lambda}, f_{D}=\frac{2\left(\nu \sin \theta_{t}+v_{t}\right) T}{\lambda}, f_{s, i}=\frac{d_{R} \sin \theta_{i}}{\lambda}, \gamma=\frac{d_{T}}{d_{R}}$, and $\beta=\frac{2 v T}{d_{R}}$, then (12) can be rewritten as

$$
\begin{aligned}
\mathbf{y}_{n, l}= & \sum_{m=0}^{M-1} \rho_{t} \mathbf{s}_{m}^{T} e^{j 2 \pi\left(n f_{s}+m \gamma f_{s}+f_{D} l\right)} \\
& +\sum_{i=0}^{N_{C}-1 M-1} \sum_{m=0} \rho_{i} \mathbf{s}_{m}^{T} e^{j 2 \pi\left(n f_{s, i}+m \gamma f_{s, i}+\beta f_{s, i} l\right)}+\mathbf{z}_{n, l} \\
= & \rho_{t} e^{j 2 \pi\left(n f_{s}+f_{D} l\right)} \mathbf{b}^{T} \mathbf{S}+\sum_{i=0}^{N_{C}-1} \rho_{i} e^{j 2 \pi\left(n f_{s, i}+\beta f_{s, i} l\right)} \mathbf{b}_{i}^{T} \mathbf{S} \\
& +\mathbf{z}_{n, l}
\end{aligned}
$$

where $\mathbf{b}=\left[1, e^{j 2 \pi \gamma f_{s}}, \ldots, e^{j 2 \pi(M-1) \gamma f_{s}}\right]^{T}$ and $\mathbf{b}_{i}=\left[1, e^{j 2 \pi \gamma f_{s, i}}\right.$, $\ldots, e^{\left.j 2 \pi(M-1) \gamma f_{s, i}\right]^{T}}$ denote, respectively, the transmitting steering vectors for the target and the clutter 
patch at $\theta_{i} . S=\left[s_{1}, s_{2}, \ldots, s_{M}\right]^{T}$ represents the waveform matrix in each PRI. For the $l$ th PRI, the data received by all the receiving elements can be expressed as

$$
\mathbf{Y}_{l}=\rho_{t} e^{j 2 \pi f_{D} l} \mathbf{a b}^{T} \mathbf{S}+\sum_{i=0}^{N_{C}-1} \rho_{i} e^{j 2 \pi \beta f_{s, l} l} \mathbf{a}_{i} \mathbf{b}_{i}^{T} \mathbf{S}+\mathbf{Z}_{l}
$$

where $\mathbf{Z}_{l}=\left[\mathbf{z}_{1, l}^{T}, \mathbf{z}_{2, l}^{T}, \ldots, \mathbf{z}_{N, l}^{T}\right]^{T} \in \mathbb{C}^{N \times K}, \mathbf{a}=\left[1, e^{j 2 \pi f_{s}}, \ldots\right.$, $\left.e^{j 2 \pi(N-1) f_{s}}\right]^{T}$, and $\mathbf{a}_{i}=\left[1, e^{j 2 \pi f_{s, i}}, \ldots, e^{j 2 \pi(N-1) f_{s, i}}\right]^{T}$ denote the receiving steering vectors for the target and the clutter patch at $\theta_{i}$, respectively. According to [5], we can assume that the columns of $\mathbf{Z}_{1}$ are independent and identically distributed circularly symmetric complex Gaussian random vectors with mean zero and an unknown covariance $Q \in \mathbb{C}^{N \times N}$.

To obtain the sufficient statistics for STAP signal processing, we can employ $S^{H}\left(S S^{H}\right)^{-1 / 2}$ as the matched filter bank [5], the output of which can be illustrated as

$$
\widetilde{\mathbf{Y}}_{l}=\rho_{t} e^{j 2 \pi f_{D} l} \mathbf{a b}^{T} \mathbf{R}_{\mathrm{s}}^{1 / 2}+\sum_{i=0}^{N_{C}-1} \rho_{\mathrm{i}} \mathrm{e}^{j 2 \pi \beta f_{s, i} l} \mathbf{a}_{i} \mathbf{b}_{i}^{T} \mathbf{R}_{\mathrm{s}}^{1 / 2}+\widetilde{\mathbf{Z}}_{l}
$$

where $\widetilde{\mathbf{Y}}_{l}=\mathbf{Y}_{l} \mathbf{S}^{H}\left(\mathbf{S S}^{H}\right)^{-1 / 2}, \quad \widetilde{\mathbf{Z}}_{l}=\mathbf{Z}_{l} \mathbf{S}^{H}\left(\mathbf{S S}^{H}\right)^{-1 / 2}, R_{S}=$ $S S^{H}$ denotes the WCM, and $(\cdot)^{1 / 2}$ represents the square root of a certain matrix [20]. The output of the matched filter can be stacked in a $M N \times 1$ vector as

$$
\begin{aligned}
\widetilde{\mathbf{y}}_{l}= & \rho_{t} e^{j 2 \pi f_{D} l}\left(\mathbf{R}_{S}^{T^{1 / 2}} \otimes \mathbf{I}_{N}\right)(\mathbf{b} \otimes \mathbf{a}) \\
& +\sum_{i=0}^{N_{C}-1} \rho_{i} e^{j 2 \pi \beta f_{s, i} l}\left(\mathbf{R}_{\mathrm{S}}^{T^{1 / 2}} \otimes \mathbf{I}_{N}\right)\left(\mathbf{b}_{i} \otimes \mathbf{a}_{i}\right) \\
& +\operatorname{vec}\left(\widetilde{\mathbf{Z}}_{l}\right),
\end{aligned}
$$

where $\widetilde{\mathbf{y}}_{l}=\operatorname{vec}\left(\widetilde{\mathbf{Y}}_{l}\right), \mathbf{I}_{\mathrm{N}}$ denotes the $N \times N$ identity matrix. Now we can obtain the total space-time snapshots as

$$
\mathcal{X}_{C}=\left[\widetilde{\mathbf{y}}_{1}^{T}, \widetilde{\mathbf{y}}_{1}^{T}, \ldots, \widetilde{\mathbf{y}}_{L}^{T}\right]^{T} \in \mathbb{C}^{\mathrm{NML} \times 1} .
$$

Substituting (16) into (17) results in

$$
\begin{aligned}
\mathcal{X}_{C}= & \rho_{t} \mathbf{u}_{D} \otimes\left(\left(\mathbf{R}_{\mathrm{S}}^{T^{1 / 2}} \otimes \mathbf{I}_{N}\right)(\mathbf{b} \otimes \mathbf{a})\right) \\
& +\sum_{i=0}^{N_{C}-1} \rho_{i} \mathbf{u}_{D, i} \otimes\left(\left(\mathbf{R}_{\mathrm{S}}^{T^{1} / 2} \otimes \mathbf{I}_{N}\right)\left(\mathbf{b}_{i} \otimes \mathbf{a}_{i}\right)\right) \\
& +\mathbf{1}_{L} \otimes \operatorname{vec}\left(\widetilde{\mathbf{Z}}_{l}\right),
\end{aligned}
$$

where $\mathbf{u}_{D}=\left[1, e^{j 2 \pi f_{D}}, \ldots, e^{j 2 \pi(L-1) f_{D}}\right]^{T}$ and $\mathbf{u}_{D, i}=\left[1, e^{j 2 \pi f_{D}}\right.$, $i, \ldots, e^{\left.j 2 \pi(L-1) f_{D, i}\right]^{T}}$ denote the Doppler steering vectors for the target and the clutter patch at $\theta_{i}$, respectively. $\mathbf{1}_{L}$ represents the $L \times 1$ vector with all elements being ones. Based on the fact that $(A B) \otimes(C D)=(A \otimes B)(C \otimes D)$, (18) can be recast as

$$
\begin{aligned}
\mathcal{X}_{C}= & \rho_{t}\left(\mathbf{I}_{L} \otimes \mathbf{R}_{S}^{T^{1} / 2} \otimes \mathbf{I}_{N}\right)\left(\mathbf{u}_{D} \otimes \mathbf{b} \otimes \mathbf{a}\right) \\
& +\sum_{i=0}^{N_{C}-1} \rho_{i}\left(\mathbf{I}_{L} \otimes \mathbf{R}_{S}^{T^{1} / 2} \otimes \mathbf{I}_{N}\right)\left(\mathbf{u}_{D, i} \otimes \mathbf{b}_{i} \otimes \mathbf{a}_{i}\right) \\
& +\mathbf{1}_{L} \otimes \operatorname{vec}\left(\widetilde{\mathbf{Z}}_{l}\right) \\
= & \rho_{t}\left(\mathbf{I}_{L} \otimes \mathbf{R}_{S}^{T^{1} / 2} \otimes \mathbf{I}_{N}\right)\left(\mathbf{u}_{D} \otimes \mathbf{b} \otimes \mathbf{a}\right) \\
& +\left(\mathbf{I}_{L} \otimes \mathbf{R}_{S}^{T^{1} / 2} \otimes \mathbf{I}_{N}\right) \sum_{i=0}^{N_{C}-1} \rho_{i}\left(\mathbf{u}_{D, i} \otimes \mathbf{b}_{i} \otimes \mathbf{a}_{i}\right) \\
& +\mathbf{1}_{L} \otimes \operatorname{vec}\left(\widetilde{\mathbf{Z}}_{l}\right) .
\end{aligned}
$$

For the optimum MIMO-STAP processor, the output SINR can be expressed as (see e.g., [15])

$$
\begin{aligned}
\mathrm{SINR}= & \left|\rho_{t}\right|^{2}\left[\left(\mathbf{I}_{L} \otimes \mathbf{R}_{S}^{T^{1} / 2} \otimes \mathbf{I}_{N}\right)\left(\mathbf{u}_{D} \otimes \mathbf{b} \otimes \mathbf{a}\right)\right]^{H} \\
& \times \mathbf{R}_{i+n}^{-1}\left[\left(\mathbf{I}_{L} \otimes \mathbf{R}_{S}^{T^{1} / 2} \otimes \mathbf{I}_{N}\right)\left(\mathbf{u}_{D} \otimes \mathbf{b} \otimes \mathbf{a}\right)\right],
\end{aligned}
$$

where

$$
\begin{aligned}
\mathbf{R}_{i+n}=E & {\left[(\mathbf{i}+\mathbf{n})(\mathbf{i}+\mathbf{n})^{H}\right] } \\
=\mathrm{E} & {\left[\left(\left(\mathbf{I}_{L} \otimes \mathbf{R}_{\mathrm{S}}^{T^{1 / 2}} \otimes \mathbf{I}_{N}\right) \sum_{i=0}^{N_{C}-1} \rho_{i}\left(\mathbf{u}_{D, i} \otimes \mathbf{b}_{i} \otimes \mathbf{a}_{i}\right)\right.\right.} \\
& \left.+\mathbf{1}_{L} \otimes \operatorname{vec}\left(\widetilde{\mathbf{Z}}_{l}\right)\right) \\
& \times\left(\left(\mathbf{I}_{L} \otimes \mathbf{R}_{\mathrm{S}}^{T^{1} / 2} \otimes \mathbf{I}_{N}\right) \sum_{i=0}^{N_{C}-1} \rho_{i}\left(\mathbf{u}_{D, i} \otimes \mathbf{b}_{i} \otimes \mathbf{a}_{i}\right)\right. \\
& \left.\left.+\mathbf{1}_{L} \otimes \operatorname{vec}\left(\widetilde{\mathbf{Z}}_{l}\right)\right)^{H}\right]
\end{aligned}
$$

in which $\mathbf{i}$ denotes the clutter, and $\mathbf{n}$ the interferenceplus-noise term. Under the assumption that the clutter is uncorrelated with the interference-plus-noise term, (21) can be rewritten as 


$$
\begin{aligned}
& \mathbf{R}_{i+n}=E {\left[(\mathbf{i}+\mathbf{n})(\mathbf{i}+\mathbf{n})^{H}\right] } \\
&=E {\left[\left(\left(\mathbf{I}_{L} \otimes \mathbf{R}_{\mathrm{S}}^{T^{1} / 2} \otimes \mathbf{I}_{N}\right) \sum_{i=0}^{N_{C}-1} \rho_{i}\left(\mathbf{u}_{D, i} \otimes \mathbf{b}_{i} \otimes \mathbf{a}_{i}\right)\right)\right.} \\
&\left.\times\left(\left(\mathbf{I}_{L} \otimes \mathbf{R}_{\mathrm{S}}^{T^{1 / 2}} \otimes \mathbf{I}_{N}\right) \sum_{i=0}^{N_{C}-1} \rho_{i}\left(\mathbf{u}_{D, i} \otimes \mathbf{b}_{i} \otimes \mathbf{a}_{i}\right)\right)^{H}\right] \\
&+E\left[\left(\mathbf{1}_{L} \otimes \operatorname{vec}\left(\widetilde{\mathbf{Z}}_{l}\right)\right)\left(\mathbf{1}_{L} \otimes \operatorname{vec}\left(\widetilde{\mathbf{Z}}_{l}\right)\right)^{H}\right] \\
&=\left(\mathbf{I}_{L} \otimes \mathbf{R}_{\mathrm{S}}^{T^{1 / 2}} \otimes \mathbf{I}_{N}\right) E\left[\left(\sum_{i=0}^{N_{C}-1} \rho_{i}\left(\mathbf{u}_{D, i} \otimes \mathbf{b}_{i} \otimes \mathbf{a}_{i}\right)\right)\right. \\
&\left.\times\left(\sum_{i=0}^{N_{C}-1} \rho_{i}\left(\mathbf{u}_{D, i} \otimes \mathbf{b}_{i} \otimes \mathbf{a}_{i}\right)\right)^{H}\right]\left(\mathbf{I}_{L} \otimes \mathbf{R}_{\mathrm{S}}^{T^{1} / 2} \otimes \mathbf{I}_{N}\right)^{H} \\
&+\mathbf{I}_{L} \otimes \mathbf{I}_{M} \otimes \mathbf{Q}
\end{aligned}
$$

According to [14], $\rho_{i}$ can be assumed to be identical independent Gaussian random variable with mean zero and variance denoting by $\sigma_{i}^{2}$. Hence, (22) can be rewritten as

$$
\begin{aligned}
\mathbf{R}_{i+n}= & \left(\mathbf{I}_{L} \otimes \mathbf{R}_{\mathrm{S}}^{T^{1} / 2} \otimes \mathbf{I}_{N}\right) \mathbf{V} \boldsymbol{\Xi} \mathbf{V}^{H}\left(\mathbf{I}_{L} \otimes \mathbf{R}_{\mathrm{S}}^{T^{1 / 2}} \otimes \mathbf{I}_{N}\right)^{H} \\
& +\mathbf{I}_{L} \otimes \mathbf{I}_{M} \otimes \mathbf{Q},
\end{aligned}
$$

where $\mathbf{V}=\left[\mathbf{v}_{1}, \mathbf{v}_{2}, \mathbf{v}_{N c}\right], v_{i}=u_{D, i} \otimes b_{i} \otimes a_{i}, i=1,2, \ldots, N_{C}$, and $\Xi=\operatorname{diag}\left(\sigma_{1}^{2}, \sigma_{2}^{2}, \ldots, \sigma_{N_{C}}^{2}\right)$. Substituting (23) into (20) yields

$$
\begin{aligned}
\operatorname{SINR} & =\left|\rho_{t}\right|^{2}\left(\mathbf{R}_{C S} \mathbf{v}_{t}\right)^{H}\left(\mathbf{R}_{\mathrm{CS}} \mathbf{R}_{\mathrm{C}} \mathbf{R}_{\mathrm{CS}}^{H}+\mathbf{Q}_{\mathrm{C}}\right)^{-1}\left(\mathbf{R}_{\mathrm{CS}} \mathbf{v}_{t}\right) \\
& =\left|\rho_{t}\right|^{2} \mathbf{v}_{t}^{H}\left(\mathbf{I}_{\mathrm{MNL}}+\mathbf{R}_{\mathrm{CS}}^{H} \mathbf{Q}_{\mathrm{C}}^{-1} \mathbf{R}_{\mathrm{CS}} \mathbf{R}_{\mathrm{C}}\right)^{-1} \mathbf{R}_{\mathrm{CS}}^{H} \mathbf{Q}_{\mathrm{C}}^{-1} \mathbf{R}_{\mathrm{CS}} \mathbf{v}_{t},
\end{aligned}
$$

where $v_{t}=\left(u_{D} \otimes b \otimes a\right), \quad \mathbf{R}_{C S}=\left(\mathbf{I}_{L} \otimes \mathbf{R}_{S}^{T^{1} / 2} \otimes \mathbf{I}_{N}\right), \quad R_{C}=$ $V \Xi V^{H}$, and $Q_{C}=I_{L} \otimes I_{M} \otimes Q$. Note that $R_{C} \geqslant 0[11], Q_{C}=$ $I_{L} \otimes I_{M} \otimes Q \otimes 0$ due to $Q \otimes 0$, and $R_{C S} \geqslant 0$ due to that the WCM $\mathbf{R}_{\mathbf{S}}$ is positive semidefinite generally [5]. With $Q_{C}^{-1}=I_{L} \otimes I_{M} \otimes Q^{-1}$, we can obtain

$$
\begin{aligned}
\mathbf{R}_{\mathrm{CS}}^{H} \mathbf{Q}_{\mathrm{C}}^{-1} \mathbf{R}_{\mathrm{CS}}= & \left(\mathbf{I}_{L} \otimes \mathbf{R}_{\mathrm{S}}^{T^{1} / 2} \otimes \mathbf{I}_{N}\right)^{H}\left(\mathbf{I}_{L} \otimes \mathbf{I}_{M} \otimes \mathbf{Q}^{-1}\right) \\
& \times\left(\mathbf{I}_{L} \otimes \mathbf{R}_{\mathrm{S}}^{T^{1} / 2} \otimes \mathbf{I}_{N}\right) \\
= & \mathbf{R}_{\mathrm{TS}}
\end{aligned}
$$

where $R_{T S}=I_{L} \otimes R_{S}^{T} \otimes Q^{-1}$. Note that $R_{T S} \geqslant 0$ because $Q_{C} \otimes 0$ and $R_{C S} \geqslant 0$ [18].

By substituting (25) into (24), (2) follows immediately.

\section{Appendix 2}

\section{The uncertainty model}

Similar to [21], the actual transmitting and receiving array steering vectors, as well as the Doppler vector, can be modeled as

$$
\widetilde{\mathbf{b}}=\mathbf{b}+\boldsymbol{\sigma}_{1}, \widetilde{\mathbf{a}}=a+\boldsymbol{\sigma}_{2}, \widetilde{\mathbf{u}}_{D}=\mathbf{u}_{D}+\boldsymbol{\sigma}_{3}
$$

where $\mathbf{b}$, $\mathbf{a}$, and $\mathbf{u}_{D}$ are, respectively, the corresponding presumed signal steering vectors, which are usually normalized so that $\mathbf{b}^{H} \mathbf{b}=M, \mathbf{a}^{H} \mathbf{a}=N$, and $u_{D}^{H} u_{D}=L . \sigma_{1}, \boldsymbol{\sigma}_{2}$, and $\boldsymbol{\sigma}_{3}$ are, respectively, unknown complex vectors describing errors of the transmitting and receiving array steering vectors, as well as the Doppler vector. The norms of these errors are assumed to be bounded, i.e., these vectors should belong to the following different uncertainty sets

$$
\begin{aligned}
& \mathbf{B}\left(\varepsilon_{1}\right)=\left\{\widetilde{\mathbf{b}} \mid \widetilde{\mathbf{b}}=\mathbf{b}+\boldsymbol{\sigma}_{1},\left\|\boldsymbol{\sigma}_{1}\right\| \leq \varepsilon_{1}\right\} \\
& \mathbf{A}\left(\varepsilon_{2}\right)=\left\{\widetilde{\mathbf{a}} \mid \widetilde{\mathbf{a}}=\mathbf{a}+\boldsymbol{\sigma}_{2},\left\|\boldsymbol{\sigma}_{2}\right\| \leq \varepsilon_{2}\right\}, \\
& \mathbf{U}\left(\varepsilon_{3}\right)=\left\{\widetilde{\mathbf{u}}_{D} \mid \widetilde{\mathbf{u}}_{D}=\mathbf{u}_{D}+\boldsymbol{\sigma}_{3},\left\|\boldsymbol{\sigma}_{3}\right\| \leq \varepsilon_{3}\right\}
\end{aligned}
$$

where $\|\cdot\|$ denotes the Euclidean norm. Following (26) and (27), the actual spatial-temporal vector $v{ }_{t}$ can be constructed as

$$
\begin{aligned}
\widetilde{\mathbf{v}}_{t}= & \widetilde{\mathbf{u}}_{D} \otimes \widetilde{\mathbf{b}} \otimes \widetilde{\mathbf{a}} \\
= & \left(\mathbf{u}_{D}+\boldsymbol{\sigma}_{3}\right) \otimes\left(\mathbf{b}+\boldsymbol{\sigma}_{1}\right) \otimes\left(\mathbf{a}+\boldsymbol{\sigma}_{2}\right) \\
= & \left(\mathbf{u}_{D}+\boldsymbol{\sigma}_{3}\right) \otimes\left(\mathbf{b} \otimes \mathbf{a}+\mathbf{b} \otimes \boldsymbol{\sigma}_{2}+\boldsymbol{\sigma}_{1} \otimes \mathbf{a}+\boldsymbol{\sigma}_{1} \otimes \boldsymbol{\sigma}_{2}\right) \\
= & \mathbf{u}_{D} \otimes \mathbf{b} \otimes \mathbf{a}+\mathbf{u}_{D} \otimes \mathbf{b} \otimes \boldsymbol{\sigma}_{2}+\mathbf{u}_{D} \otimes \boldsymbol{\sigma}_{1} \otimes \mathbf{a} \\
& +\mathbf{u}_{D} \otimes \boldsymbol{\sigma}_{1} \otimes \boldsymbol{\sigma}_{2}+\boldsymbol{\sigma}_{3} \otimes \mathbf{b} \otimes \mathbf{a}+\boldsymbol{\sigma}_{3} \otimes \mathbf{b} \otimes \sigma_{2} \\
& +\boldsymbol{\sigma}_{3} \otimes \boldsymbol{\sigma}_{1} \otimes \mathbf{a}+\boldsymbol{\sigma}_{3} \otimes \boldsymbol{\sigma}_{1} \otimes \boldsymbol{\sigma}_{2}
\end{aligned}
$$

With $v_{t}=\left(u_{D} \otimes b \otimes a\right)$ and (28), the spatial-temporal vector error, denoting by $\boldsymbol{\sigma}$, can be described as

$$
\begin{aligned}
\boldsymbol{\sigma}= & \mathbf{u}_{D} \otimes \mathbf{b} \otimes \boldsymbol{\sigma}_{2}+\mathbf{u}_{D} \otimes \boldsymbol{\sigma}_{1} \otimes \mathbf{a}+\mathbf{u}_{D} \otimes \boldsymbol{\sigma}_{1} \otimes \boldsymbol{\sigma}_{2} \\
& +\boldsymbol{\sigma}_{3} \otimes \mathbf{b} \otimes a+\boldsymbol{\sigma}_{3} \otimes \mathbf{b} \otimes \boldsymbol{\sigma}_{2}+\boldsymbol{\sigma}_{3} \otimes \boldsymbol{\sigma}_{1} \otimes \mathbf{a} \\
& +\boldsymbol{\sigma}_{3} \otimes \boldsymbol{\sigma}_{1} \otimes \boldsymbol{\sigma}_{2} .
\end{aligned}
$$

Following (27), the bound of the norm of $\boldsymbol{\sigma}$ can be obtained by

$$
\begin{aligned}
\|\boldsymbol{\sigma}\| \leq & \left\|\mathbf{u}_{D} \otimes \mathbf{b} \otimes \boldsymbol{\sigma}_{2}\right\|+\left\|\mathbf{u}_{D} \otimes \boldsymbol{\sigma}_{1} \otimes \mathbf{a}\right\|+\left\|\mathbf{u}_{D} \otimes \boldsymbol{\sigma}_{1} \otimes \boldsymbol{\sigma}_{2}\right\| \\
& +\left\|\boldsymbol{\sigma}_{3} \otimes \mathbf{b} \otimes \mathbf{a}\right\|+\left\|\boldsymbol{\sigma}_{3} \otimes \mathbf{b} \otimes \boldsymbol{\sigma}_{2}\right\|+\left\|\boldsymbol{\sigma}_{3} \otimes \boldsymbol{\sigma}_{1} \otimes \mathbf{a}\right\| \\
& +\left\|\boldsymbol{\sigma}_{3} \otimes \boldsymbol{\sigma}_{1} \otimes \boldsymbol{\sigma}_{2}\right\| \\
\leq & \sqrt{M} \varepsilon_{2} \varepsilon_{3}+\sqrt{N} \varepsilon_{1} \varepsilon_{3}+\sqrt{L} \varepsilon_{1} \varepsilon_{2}+\sqrt{M N} \varepsilon_{3}+\sqrt{L M} \varepsilon_{2} \\
& +\sqrt{L N} \varepsilon_{1}+\varepsilon_{1} \varepsilon_{2} \varepsilon_{3}
\end{aligned}
$$

With (28) to (30), the uncertainty set of $v^{\prime \prime}$ can be formulated as 


$$
\mathbf{V}=\left\{\widetilde{\mathbf{v}}_{t} \mid \widetilde{\mathbf{v}}_{t}=\mathbf{v}_{t}+\boldsymbol{\sigma},\|\boldsymbol{\sigma}\| \leq \varepsilon\right\},
$$

where

$$
\begin{aligned}
\varepsilon= & \sqrt{M} \varepsilon_{2} \varepsilon_{3}+\sqrt{N} \varepsilon_{1} \varepsilon_{3}+\sqrt{L} \varepsilon_{1} \varepsilon_{2}+\sqrt{M N} \varepsilon_{3} \\
& +\sqrt{L M} \varepsilon_{2}+\sqrt{L N} \varepsilon_{1}+\varepsilon_{1} \varepsilon_{2} \varepsilon_{3} .
\end{aligned}
$$

Because $|\|A\|-\|B\|| \leq\|A+B\|[20]$, (31) can equivalently be represented as

$$
\mathbf{V}=\left\{\widetilde{\mathbf{v}}_{t} \mid \eta \leq\left\|\widetilde{\mathbf{v}}_{t}\right\|\right\}
$$

where

$$
\eta=|\sqrt{\mathrm{MNL}}-\varepsilon|
$$

We make an assumption

$$
\eta \neq 0 .
$$

In other words, we rule out the possibility that the norm of the worst-case spatial-temporal vector is zero.

\section{Competing interest}

In this article, we investigate the problem of improving the worst-case detection performance of MIMO-STAP by optimizing the transmitted waveforms over the convex uncertainty consisting of the initial parameter estimation errors. By exploiting the diagonal loading method, the resultant nonlinear problem can be solved resorting to a convex relaxation that belongs to the semidefinite programming (SDP) class.

\section{Acknowledgments}

This study was sponsored in part by the NSFC under grant 60825104, 61271292, Program for Changjiang Scholars and Innovative Research Team in University under grant IRT0954, and the Major State Basic Research Development Program of China (973 Program) under grant 2010CB731903, 2011 CB707001.

\section{Author details}

${ }^{1}$ National Key Laboratory of Radar Signal Processing, Xidian University, Xi'an 710071, China. ${ }^{2}$ No.20 Research Institute of China Electronics Technology Group Corporation, Xi'an 710068, China. ${ }^{3}$ National Key Laboratory of Integrated Services Networks, Xidian University, Xi'an 710071, China.

\section{Received: 28 October 2011 Accepted: 17 December 2012}

Published: 18 March 2013

\section{References}

1. E Fishler, A Haimovich, R Blum, D Chizhik, L Cimini, R Valenzuela, MIMO radar: an idea whose time has come, in Proceedings of the IEEE Radar Conference, ed. by ( Newark, NJ, USA, 2004), pp. 71-78

2. J Li, P Stoica, MIMO radar with colocated antennas. IEEE Signal Process. Mag. 24(5), 106-114 (2007)

3. J Li, P Stoica, L Xu, W Roberts, On parameter identifiability of MIMO radar. IEEE Trans. Signal Process. Lett. 14(12), 968-971 (2007)

4. Y Haidong, L Jun, L Guisheng, Multitarget identification and localization using bistatic MIMO radar systems. EURASIP J. Adv. Signal Process. 2008, 8 (2008)

5. J Li, L Xu, P Stoica, KW Forsythe, DW Bliss, Range compression and waveform optimization for MIMO radar: a Cramer-Rao bound based study. IEEE Trans. Signal Process. 56(1), 218-232 (2008)

6. B Friedlander, Waveform design for MIMO radars. IEEE Trans. Aerospace Electron. Syst. 43(3), 1227-1238 (2007)
7. CY Chen, PP Vaidyanathan, MIMO radar waveform optimization with prior information of the extended target and clutter. IEEE Trans. Signal Process. 57(9), 3533-3544 (2009)

8. CY Chong, P Frédéric, O Jean-Philippe, L Marc, MIMO radar detection in non-Gaussian and heterogeneous clutter. IEEE J. Sel. Topics Signal Process. 4(1), 115-126 (2010)

9. Q He, NH Lehmann, RS Blum, AM Haimovich, MIMO radar moving target detection in homogeneous clutter. IEEE Trans. Aerospace Electron. Syst. 46(3), 1290-1301 (2010)

10. DW Bliss, KW Forsythe, Multiple-input multiple-output (MIMO) radar and imaging: degrees of freedom and resolution, in Proceedings of the 37th IEEE Asilomar Conference on Signals, Systems, Computers, ed. by, vol. 1st edn., 2003, pp. 54-59

11. C-Y Chen, PP Vaidyanathan, MIMO radar space-time adaptive processing using prolate spheroidal wave functions. IEEE Trans. Signal Process. 56(2), 623-635 (2008)

12. G Wang, Y Lu, Clutter rank of STAP in MIMO radar with waveform diversity. IEEE Trans. Signal Process. 58(2), 938-943 (2010)

13. JG Proakis, Digital Communications, 4th edn. (McGraw Hill, New York, 2001)

14. J Ward, Space-Time Adaptive Processing for Airborne Radar (MIT Lincoln Lab, Lexington, MA, 1994), p. 1015. Technical Report

15. R Klemn, Principles of Space-Time Adaptive Processing (IEE Press, London, UK, 2002)

16. H Cox, RM Zeskind, MH Owen, Robust adaptive beam-forming. IEEE Trans. Acoust. Speech Signal Process. 35, 1365-1376 (1987)

17. A Ben-Tal, A Nemirovski, Optimization, Lectures on Modern Convex Optimization (MPS-SIAM, Philadelphia, 2001)

18. H Lütkepohl, Handbook of Matrices (Wiley, New York, USA, 1996)

19. YC Eldar, A Ben-Tal, A Nemirovski, Robust mean-squared error estimation in the presence of model uncertainties. IEEE Trans. Signal Process. 53(1), 168-181 (2005)

20. RA Horn, CR Johnson, Matrix Analysis (Cambridge University Press, Cambridge, UK, 1985)

21. SA Vorobyov, AB Gershman, ZQ Luo, Robust adaptive beam-forming using worst-case performance optimization: a solution to the signal mismatch problem. IEEE Trans. Signal Process. 51, 313-324 (2003)

22. J Lofberg, YALMIP: a toolbox for modeling and optimization in MATLAB, in Proceedings of the CACSD Conference, Taipei, Taiwan, ed. by, 2004, pp. 284-289

23. P Stoica, J Li, X Zhu, Waveform synthesis for diversity-based transmit beampattern design. IEEE Trans. Signal Process. 56(6), 2593-2598 (2008)

doi:10.1186/1687-6180-2013-52

Cite this article as: Wang et al:: Robust waveform design for MIMO-STAP to improve the worst-case detection performance. EURASIP Journal on Advances in Signal Processing 2013 2013:52.

\section{Submit your manuscript to a SpringerOpen ${ }^{\circ}$ journal and benefit from:}

- Convenient online submission

- Rigorous peer review

- Immediate publication on acceptance

- Open access: articles freely available online

- High visibility within the field

- Retaining the copyright to your article

Submit your next manuscript at $>$ springeropen.com 